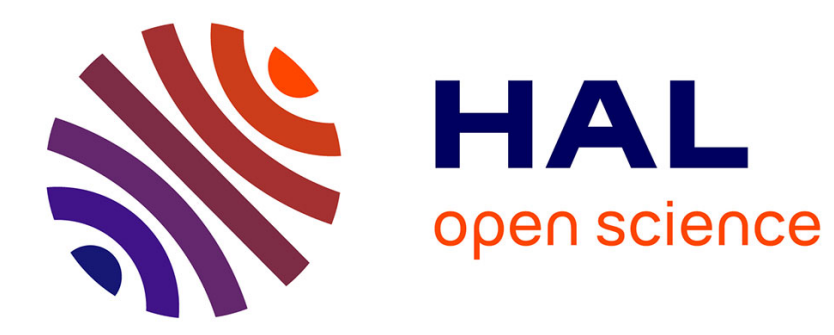

\title{
L'EMPLOI DU DISQUE D'ACKERMANN
}

R. Moreau

\section{- To cite this version:}

R. Moreau. L'EMPLOI DU DISQUE D'ACKERMANN. Le Lait, 1945, 25 (249_250), pp.320-321. hal-00927913

\section{HAL Id: hal-00927913 \\ https://hal.science/hal-00927913}

Submitted on 1 Jan 1945

HAL is a multi-disciplinary open access archive for the deposit and dissemination of scientific research documents, whether they are published or not. The documents may come from teaching and research institutions in France or abroad, or from public or private research centers.
L'archive ouverte pluridisciplinaire HAL, est destinée au dépôt et à la diffusion de documents scientifiques de niveau recherche, publiés ou non, émanant des établissements d'enseignement et de recherche français ou étrangers, des laboratoires publics ou privés. 
des réactions pendant 30 minutes pour le Dupouy et 3 ou 4 heures pour le Schardinger, temps au bout desquels ces réactions peuvent être considérées comme définitivement négatives;

$6^{\circ}$ La réaction de Schardinger sera conduite de la manière suivante :

A $10 \mathrm{~cm}^{3}$ de lait introduits dans un tube à essai de faible diamètre, ajouter $0 \mathrm{~cm}^{3} 5$ de réactif de Schardinger. Boucher le tube et le porter au bain-marie à $50^{\circ}$. Examiner le tube au bout de quatre heures. Si le tube est resté bleu dans toute sa longueur la réaction est négative et la pasteurisation suffisante. Si, au bout de ce temps, on observe une décoloration partielle, même minime ( $a u$ fond du tube) la réaction doit être considérée comme positive et la pasteurisation déclarée insuffisante ;

$7^{\circ}$ La réaction de Schardinger, appliquée de la même manière, est particulièrement recommandable pour le contrôle de la pasteurisation des crèmes.

(Signalons que la partie expérimentale de ce travail a été effectuée avec la collaboration de M. LIGNAC du laboratoire de Bactériologie de la S. A. F. R. que nous remereions partieulièrement pour le soin qu'il a apporté dans l'exécution des essais.)

\section{L'EMPLOI DU DISQUE D'ACKERMANN}

$$
\text { par }
$$

\section{R. MOREAU}

La détermination de l'extrait sec dégraissé d'un lait, par dessiccation à l'étuve et extraction à l'éther de la matière grasse, si elle ne constitue pas en principe une opération compliquée, exige cependant de la part de l'opérateur une habitude des manipulations, certaines précautions et un temps déjà important.

Beaucoup de laboratoires industriels ne disposent pas du personnel "ad hoc » et, dans ce cas, le chiffre trouvé risque de présenter une approximation moins grande que celui qui serait obtenu à l'aide des tables de Fleishmann ou du disque d'Ackermann. Encore faut-il que tables ou disque soient employés correctement.

Or, dans la plupart des laboratoires, le chimiste analyste se contente, en ce qui concerne la matière grasse, de reporter sur le disque d'Ackermann le chiffre indiqué par l'échelle graduée du butyromètre Gerber et de lire sûr le disque, en fonction de la densité, l'extrait sec correspondant, en le considérant, à tort, comme se rapportant à un litre de lait. 
Or, le butyromètre Gerber, contrairement à la notation \%, indique une richesse au litre et non au kilogramme (1) et c'est cependant cette dernière qui doit être transposée sur le disque d'Ackermann qui donne l'extrait sec par kilogramme de lait et non par litre de lait.

Examinons, à l'aide d'un exemple, l'erreur commise. Considérons un lait ayant 1.032 grammes comme poids spécifique et contenant 35 grammes de matière grasse par litre.

En employant le processus erronné habituel, nous aurons comme extrait sec dégraissé :

$124,8-35=89,8$ alors que G. Thieulin et R. Vurllaume, dans leur ouvrage intitulé «Eléments pratiques d'analyse et d'inspection du lait " admettent le chiffre de 92 .

La méthode correcte, malheureusement rarement employée, consistera à rechercher l'extrait sec par $\mathrm{kg}$. en fonction de la densité et du taux de matière grasse par kilogramme, puis à multiplier le résultat obtenu par la densité.

Dans le cas considéré, nous aurons :

Taux de matière grasse par $\mathrm{kg} .=\frac{35 \times 1.000}{1.032}=33,9$

Le disque d'Ackermann nous donnera comme extráit sec 123,4 par kilogramme, e'est-à-dire $123,4 \times 1,032=127 \mathrm{gr}, 3 \mathrm{par}$ litre,

d'où nous déduirons, comme extrait sec dégraissé, par litre : - $127,3-35=92,3$ : chiffre voisin du chiffre de G. Thinulin et R. Vuillaume.

- Il est regrettable que dans l'industrie laitière, certains problèmes pratiques n'aient pas été suffisamment approfondis par les techniciens des laboratoires. Il en résulte que les méthodes employées par les industriesl fromagers et beurriers sont parfois entachées d'erreurs qui, au cours d'une journée, sont appelées à se répéter de nombreuses fois.

(1) Les travaux de Kosstier et Lortschir, Brioux, Pien, et les récentes expé. riences faites à ma demande par le Professeur ScнмidT, maître de conférences à la Faculté des Sciences de Rennes, l'ont établi d'une façon indiscutable.

(2) Tous les butyromètres donnant un résultat $\alpha$ au litre » et utilisés couramment en France rendent évidemment cette correction obligatoire. 